\title{
Contemporary temperature-driven divergence in a Nordic freshwater fish under conditions commonly thought to hinder adaptation
}

\author{
Kathryn D Kavanagh ${ }^{1,6+}$, Thrond O Haugen ${ }^{2,3,4^{*}+}$, Finn Gregersen ${ }^{3}$, Jukka Jernvall ${ }^{1,5}$, L Asbjørn Vøllestad ${ }^{3}$
}

\begin{abstract}
Background: Evaluating the limits of adaptation to temperature is important given the IPCC-predicted rise in global temperatures. The rate and scope of evolutionary adaptation can be limited by low genetic diversity, gene flow, and costs associated with adaptive change. Freshwater organisms are physically confined to lakes and rivers, and must therefore deal directly with climate variation and change. In this study, we take advantage of a system characterised by low genetic variation, small population size, gene flow and between-trait trade-offs to study how such conditions affect the ability of a freshwater fish to adapt to climate change. We test for genetically-based differences in developmental traits indicating local adaptation, by conducting a common-garden experiment using embryos and larvae from replicate pairs of sympatric grayling demes that spawn and develop in natural cold and warm water, respectively. These demes have common ancestors from a colonization event 22 generations ago. Consequently, we explore if diversification may occur under severely constraining conditions.

Results: We found evidence for divergence in ontogenetic rates. The divergence pattern followed adaptation predictions as cold-deme individuals displayed higher growth rates and yolk conversion efficiency than warmdeme individuals at the same temperature. The cold-deme embryos had a higher rate of muscle mass development. Most of the growth- and development differences occurred prior to hatch. The divergence was probably not caused by genetic drift as there was a strong degree of parallelism in the divergence pattern and because phenotypic differentiation $\left(\mathrm{Q}_{\mathrm{ST}}\right)$ was larger than estimated genetic drift levels (microsatellite $\mathrm{F}_{\mathrm{ST}_{\mathrm{S}}}$ ) between demes from different temperature groups. We also document that these particular grayling populations cannot develop successfully at temperatures above $12^{\circ} \mathrm{C}$, whereas other European populations can, and that increasing the muscle mass development rate comes at the cost of some skeletal trait development rates.

Conclusions: This study shows that genetically based phenotypic divergence can prevail even under conditions of low genetic variation and ongoing gene flow. Furthermore, population-specific maximum development temperatures along with musculoskeletal developmental trade-offs may constrain adaptation.
\end{abstract}

\section{Background}

Evolutionary change in a population can be very fast, with measurable genetic change occurring over only a few generations [1]. However, the ability of animals to evolve to keep pace with rapid climate change remains enigmatic $[3,4]$. There are a number of factors that might constrain or impede such rapid adaptation.

\footnotetext{
* Correspondence: thrond.haugen@hihm.no

+ Contributed equally

${ }^{2}$ Norwegian Institute for Water Research, Gaustadalléen 21, NO-0349 Oslo,

Norway

Full list of author information is available at the end of the article
}

Firstly, low genetic diversity, where small or homogeneous populations lack the genetic "raw material" for adaptation is commonly assumed to constrain adaptation [5]. Secondly, ongoing gene flow is assumed to strongly constrain adaptation, where local adaptations, especially those arising in "sink" demes, are swamped by alleles from interbreeding (source) demes adapted to other environments [6,7]. Various quantitative genetic models have attempted to predict the constraints caused by gene flow under migration - selection balance $[8,9]$ but clearly more empirical tests are needed. Thirdly, genetic drift may strongly constrain adaptive evolution,

\section{(Ciomed Central}


where small populations suffer from maladaptive genetic differentiation due to influence from random processes [10]. Lastly, there may be costs to adaptation, where agonistic pleiotropy [7] or developmental integration [11] limits adaptation of any given trait because of its interaction with other traits during development.

Demonstrating that natural populations have adapted to climate change or novel habitats is not an easy task [12]. This will require data that either shows, or from which it can be inferred, that traits under study have undergone selection. Also, this selection process should be linked, either directly or indirectly, via known environment and organism interaction mechanisms, to climate change or novel habitat characteristics. Finally, the change in trait values has to be demonstrated to have a genetic basis that is adaptive. A recent review concluded that even though the evidence for climate- and habitat change-driven phenotypic responses in wild populations is indisputable, it is less clear what underlying mechanisms are causing these changes [12]. Indeed, many responses perceived as adaptations to changing environmental conditions could be environmental-induced plasticity. Also, plasticity itself may evolve. Lande (2009) [13] suggested that adaptation actually may occur in two phases, the first being rapid evolution of increased plasticity in the new environment allowing the mean phenotype to quickly approach the new optimum. This first phase is then followed by a slower genetic assimilation.

In this study, we examined the ability of a freshwater fish to adapt to conditions relevant to climate change under circumstances that, theoretically, should limit adaptation. In the early 1880 s, a man-made connection to the upper reaches of the river Gudbrandsdalslågen, Norway, allowed grayling (Thymallus thymallus) to colonize the mountain lake Lesjaskogsvatnet [14] (Figure 1). Later dam constructions severely reduced this opportunity for further invasions of grayling, but grayling from Lesjaskogsvatnet may disperse back to the river system. The exact number of founders is unknown, but it is likely to have been a limited number due to the poor grayling habitats in the upper reaches of the river. Furthermore, analyses of microsatellite DNA have revealed that the source population possesses very low levels of neutral genetic variation [15]. When grayling colonize new lake/river systems, their homing behaviour allows rapid structuring into reproductively more or less isolated demes [16]. The Lesjaskogsvatnet population is currently divided into ca. 20 demes spawning in different tributaries [17]. Microsatellite data from Lesjaskogsvatnet shows significant differentiation among many of the grayling demes in the lake with a significant isolation by distance population structure [18], but the population structure is unstable with the underlying isolation by distance structure in some years being swamped by gene flow [19]. All demes spawning in the tributaries also show clear signatures of recent bottlenecks, but based on the available evidence it is not clear if this is a signal from the original invasion of the lake or more recent independent events $[18,19]$.

Lesjaskogsvatnet is an elongated lake and consists of a shady and a sunny side because of high surrounding mountains. Demes spawning in sunny-side tributaries spawn up to four weeks earlier and their eggs and larvae have a considerably higher development temperature as water temperatures rise much faster than in shady side tributaries (Table 1 and 2), thus providing natural replicates of "warm" (sunny) and "cold" (shady) tributaries (Figure 1, Additional file 1). The young grayling stay in the tributaries for 2-3 months (allopatric phase), after which they mix with other demes within the lake (sympatric phase). Once in the lake, the juveniles from both warm and cold demes will compete over the same food resources striving to access enough food to build up sufficient resources for surviving their first 6-7 month winter, when feeding opportunities are strongly reduced. For juvenile freshwater fish in temperate areas, large body size, and hence larger energy reserves has repeatedly been shown to increase accession of food and winter survival [20]. Being born weeks later than warmdeme embryos, cold-deme embryos face a tremendous time-constraint as they have shorter time available for attaining a certain size and/or energy level to make it through the first winter. On top of that, the cold-deme embryos need to grow and develop faster under colder conditions (Table 2).

To test for genetic differentiation among demes in developmental traits, we conducted a common-garden experiment using embryos and larvae from two cold and two warm demes (Figure 1) exposed to $8^{\circ} \mathrm{C}$ and $12^{\circ} \mathrm{C}$ water temperature (see Methods section). This setup allows for testing for genetic differences among demes exposed to the two temperatures, but does not explicitly test for local adaptation. We chose $8^{\circ} \mathrm{C}$ as the commongarden temperature since embryos from all demes would experience this temperature in nature soon after fertilization. The $12^{\circ} \mathrm{C}$ treatment level was chosen to test the effect of a high incubation temperature within a potential range that may result from global warming. Since growth rate in fishes, as measured in length or mass, represents primarily growth in the musculoskeletal system [21], and previous studies have reported physiological or performance trade-offs in growth-rate evolved populations [22-24], we investigated differences in musculoskeletal developmental rate evolution in more detail. We analyzed both muscle-fibre development and skeletal ossification in the four demes. The common-garden study design employed in this study allows for assessment of parallelism in divergence for pairs of demes 


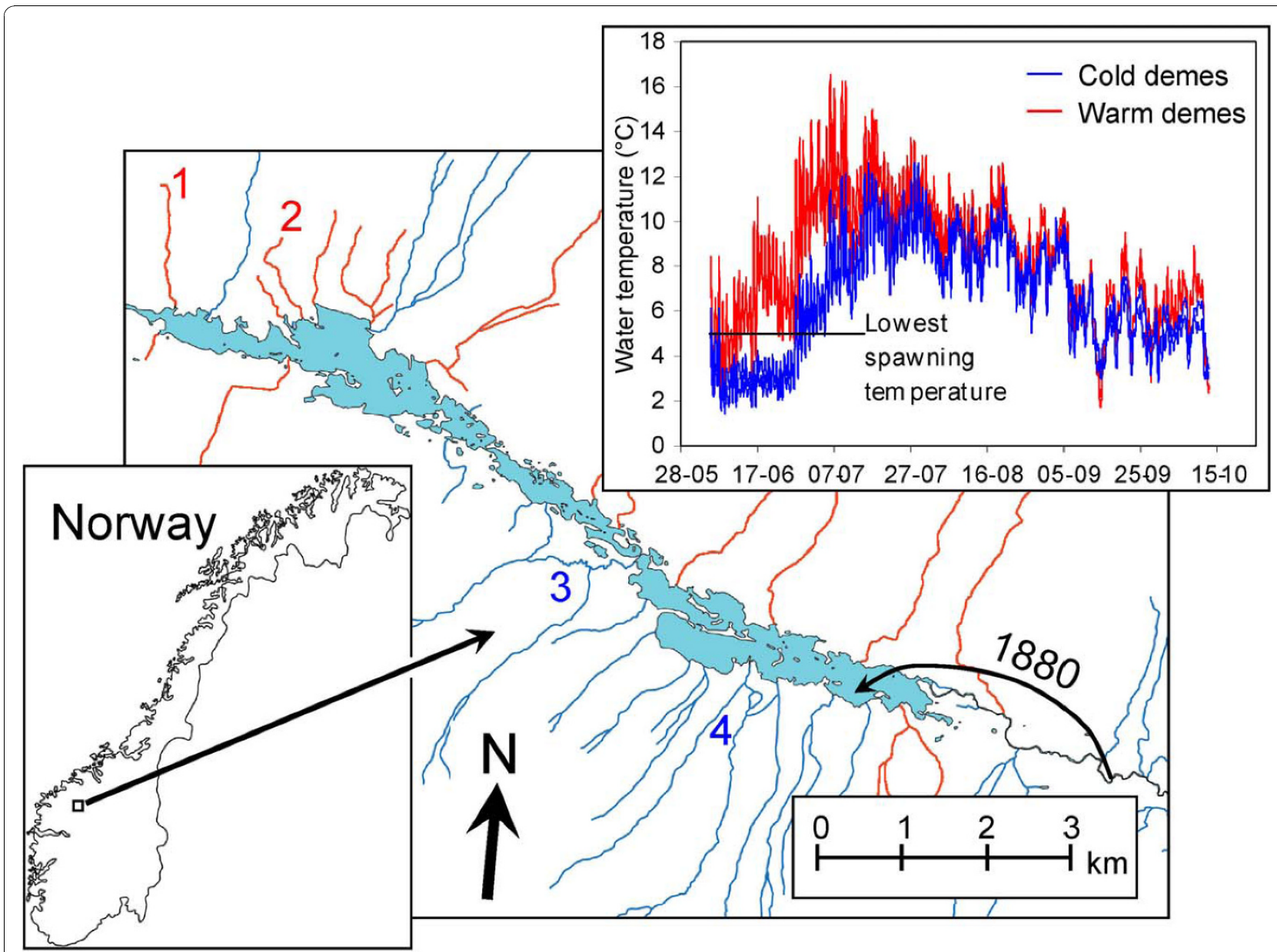

Figure 1 Study system and stream temperature profiles. Map showing the location of the study site as well as the 2005 temperature profiles for the four demes involved in the common-garden experiment. Numbers attached to tributaries display the location of the demes (warm demes red numbers, cold demes blue numbers). The 1880 labelling refers to the year of colonization of the lake.

belonging to the same temperature regime. In order to evaluate the relative role of natural selection and genetic drift to observed phenotypic differentiation, we used information about microsatellite-based $\mathrm{F}_{\mathrm{ST}}$ as a measure of drift-generated genetic differentiation. These estimates were compared with the analogous trait-wise $\mathrm{Q}_{\mathrm{ST}}$-estimates measuring genetic differentiation in phenotypic traits, where $\mathrm{Q}_{\mathrm{ST}}>\mathrm{F}_{\mathrm{ST}}$ suggest differentiation due to divergent selection and $\mathrm{Q}_{\mathrm{ST}}<\mathrm{F}_{\mathrm{ST}}$ suggest stabilizing selection [25]. By combining information on 1) degree of parallelism in trait expression between demes from same temperature group with 2) the degree of congruence between the observed and expected direction of trait differentiation and 3) pair wise $\mathrm{Q}_{\mathrm{ST}} \mathrm{vs} \mathrm{F}_{\mathrm{ST}}$ comparisons we are able to draw inferences about signatures of local adaptation.

Table 1 Environmental characteristics for the ancestral (Gudbrandsdalslågen) and colonized systems (warm streams in bold and cold in italics)

\begin{tabular}{lllc}
\hline Location (Figure 1) & Mean width $(\mathbf{m})$ & Mean discharge $\left(\mathbf{m}^{\mathbf{3}} / \mathbf{s e c}\right)$ & Mean water temperature during spawning period $\left({ }^{\circ} \mathbf{C}\right){ }^{\mathbf{1}}$ \\
\hline Gudbrandsdalslågen & 14.3 & $10.1 \pm 15.3$ & $6.3 \pm 1.4(1992-2001)$ \\
Bella (1) & $\mathbf{1 . 3}$ & $\mathbf{0 . 0 9}$ & $\mathbf{6 . 1} \pm \mathbf{0 . 7}(\mathbf{2 0 0 5 - 2 0 0 6 )}$ \\
Søre Skotte (2) & $\mathbf{0 . 7}$ & $\mathbf{0 . 0 2}$ & $\mathbf{5 . 8} \pm \mathbf{1 . 7}(\mathbf{2 0 0 3 - 2 0 0 7 )}$ \\
Hyrjon (3) & 5.0 & 0.25 & $3.2 \pm 1.1(1996-2007)$ \\
Valåa (4) & 5.8 & 0.42 & $2.9 \pm 0.9(1996-2007)$ \\
\hline
\end{tabular}

${ }^{1} 01$ June-10 July (years covered are provided in parenthesis) 
Table 2 Estimated development temperature sums (D). $t_{\min }$ refers to the date at which the stream water temperature exceeds $4^{\circ} \mathrm{C}$ for the first time during a given year.

\begin{tabular}{|c|c|c|c|c|}
\hline \multirow[b]{2}{*}{ Development period } & \multicolumn{2}{|c|}{$D$ (mean \pm s.e.) ${ }^{\circ} \mathrm{C}$} & \multirow[b]{2}{*}{$F^{1}$} & \multirow[b]{2}{*}{$P$} \\
\hline & Warm demes & Cold demes & & \\
\hline$t_{\min }+2$ weeks & $113.3 \pm 2.7$ & $91.1 \pm 3.1$ & 22.10 & $<0.0001$ \\
\hline$t_{\min }+4$ weeks & $242.2 \pm 4.2$ & $220.0 \pm 4.5$ & 10.64 & 0.0033 \\
\hline$t_{\min }+6$ weeks & $394.0 \pm 5.1$ & $371.1 \pm 5.4$ & 5.04 & 0.0342 \\
\hline
\end{tabular}

${ }^{1} \mathrm{df}=1,24$ for all tests. One-way anova.

\section{Results}

At $12^{\circ} \mathrm{C}$, no embryos survived past 5 days post hatch, while at $8^{\circ} \mathrm{C}$ many embryos survived to 30 days post hatch (when the experiment was terminated).

\section{Individual growth processes}

While egg diameter and dry weights were not different among demes $\left(F_{3,24}=1.93, P=0.15\right)$, a comparison of mean dry weights of fertilized eggs, pre-hatch yolk and embryos (weighed separately), and pre-feeding larvae revealed that the cold deme embryos grew faster and absorbed yolk faster than warm deme embryos (Figure 2A, B). Yolk conversion efficiencies (i.e., how efficiently yolk mass was transformed to body mass) were significantly and consistently greater in cold demes than in warm demes (Figure 2C). Notochord length $(N L)$ measurements from digital images of all daily samples from each deme ( $n=6-10 /$ deme/day) confirmed that size-at-age differences were found throughout larval development, replicated in both cold demes and warm demes (Figure 2D). A generalized additive model fitting size-at-age as a function of days post fertilization $(D P F)$ and temperature group, revealed that all demes had parallel growth trajectories (i.e., little support of a $D P F^{*}$ deme model as AIC was 67 levels larger than the $D P F+$ deme model) where cold-deme embryos started out as $1.00 \mathrm{~mm}$ longer (s.e.m $=0.11, t=-8.99, P<$ 0.0001 , Additional file 2) at $21 D P F$ and ended up as $1.00 \mathrm{~mm}$ longer after 13 days.

\section{Developmental trait trade-offs}

In histological cross-sections of muscle made at a homologous point (the anus), we found that fastergrowing cold deme individuals had a significantly larger average muscle fibre area at a given age and size (Least Square Mean $(L S M) \pm 2$ s.e.m. from generalized linear model $(\mathrm{GLM}): N L=14 \mathrm{~mm}: \mathrm{LSM}_{\text {cold }}=142.6 \pm 12.4$ $\mu \mathrm{m}^{2}, \mathrm{LSM}_{\text {warm }}=115.4 \pm 10.3 \mu \mathrm{m}^{2}$ ) (Figure 3). In contrast, rate of skeletal developmental was delayed in cold deme fish when compared with similar-sized fish from warm demes. Both axial and cranial skeletal features developed faster in cold demes (all 9 traits had lower group effect coefficients for warm demes, GLM-derived

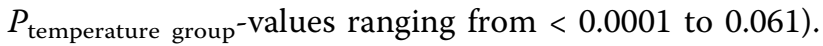
Hence, cold deme individuals reached a certain trait expression probability earlier than warm-deme individuals. However, in 7 out of 9 traits (GLM-derived

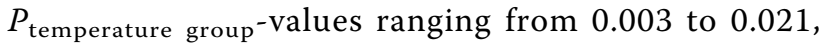
Additional file 2), the skeletal development did not "keep up" proportionately with notochord length in the cold demes (Figure 4).

\section{Qst vs Fst}

Pair-wise differences in analogous $\mathrm{F}_{\mathrm{ST}}$ and $\mathrm{Q}_{\mathrm{ST}}$ values are displayed, for three traits, in Additional file 3. In total, $88 \%(28 / 32)$ of the warm vs cold deme pair wise comparisons are consistent with divergent natural selection and $12 \%$ could not be distinguished from the pattern expected under genetic drift. For comparisons between demes within the same temperature group only $19 \%(3 / 16)$ of the comparisons indicated divergent selection, whereas $81 \%$ could be attributed to convergent evolution or drift. Because 5 out of $6 \mathrm{~F}_{\mathrm{ST}}$ values had confidence bounds that overlapped with zero, inferences on eventual convergent evolution (i.e., $\mathrm{F}_{\mathrm{ST}}>\mathrm{Q}_{\mathrm{ST}}$ ) could not be made.

\section{Discussion}

This study provides compelling evidence that individual growth- and developmental rate and efficiency can evolve over fewer than 22 generations under conditions widely theorized to impede local adaptation. In particular, the degree of parallelism in the direction of divergence for most of the traits investigated reveals compelling signals of adaptation-driven differentiation. Cold deme individuals were shown (with rare exception) to consistently have a more efficient yolk-to-body-mass conversion efficiency, a higher somatic growth rate and a higher skeletal- and muscle mass developmental rate compared to warm-deme individuals. All of this is in accordance with predictions on expected direction of divergence caused by temperature differences between deme habitats. Since some divergence has take place and also since this divergence is according to the predicted direction of trait evolution, we conclude they are not severely constrained or limited in their adaptation to temperature differences - even under conditions with substantial gene flow. However, we cannot rule out that they may have been constrained below what would be the optimum divergence. One should bear in mind the spatial scale for this particular study system - the demes are separated by just $2-6 \mathrm{~km}$ during the reproductive period. As a consequence, any spawning site can easily be accessed by any adult grayling individual in the lake during just one day (e.g., a $30 \mathrm{~cm}$ individual can at a cruising speed of 0.25 body lengths per second reach 


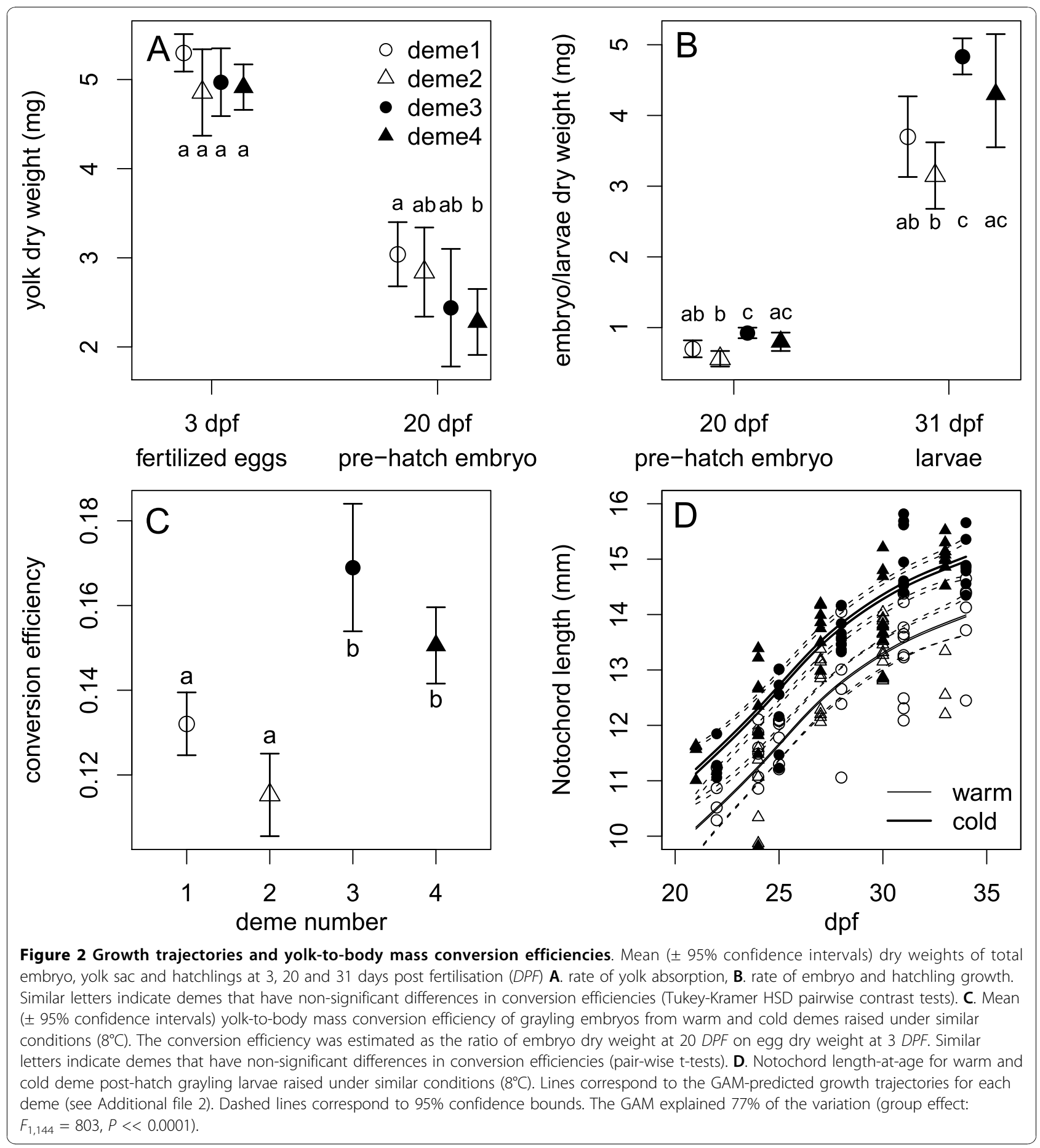

more than $6 \mathrm{~km}$ during a day). Still, we know from previous studies that local adaptation can occur within such spatio-temporal scales [26-28]. In fact, our findings of temperature-related divergence of developmental and ontogenetic timing traits are very much in agreement with findings in other salmonid systems with recent origin. Very similar to our findings, in a system very comparable to our grayling system, Hendry et al. [29] found that development rate was fastest for progeny of late spawning sockeye salmon (Oncorhynchus nerka) demes in Lake Washington. Further, yolk conversion efficiency was related to natural incubation temperatures. This adaptive divergence pattern had arisen in only 9-14 generations, within the same lake system with demes separated by less than $10 \mathrm{~km}$. Similarly, Kinnison et al [31] found that early-life growth rates differed between 


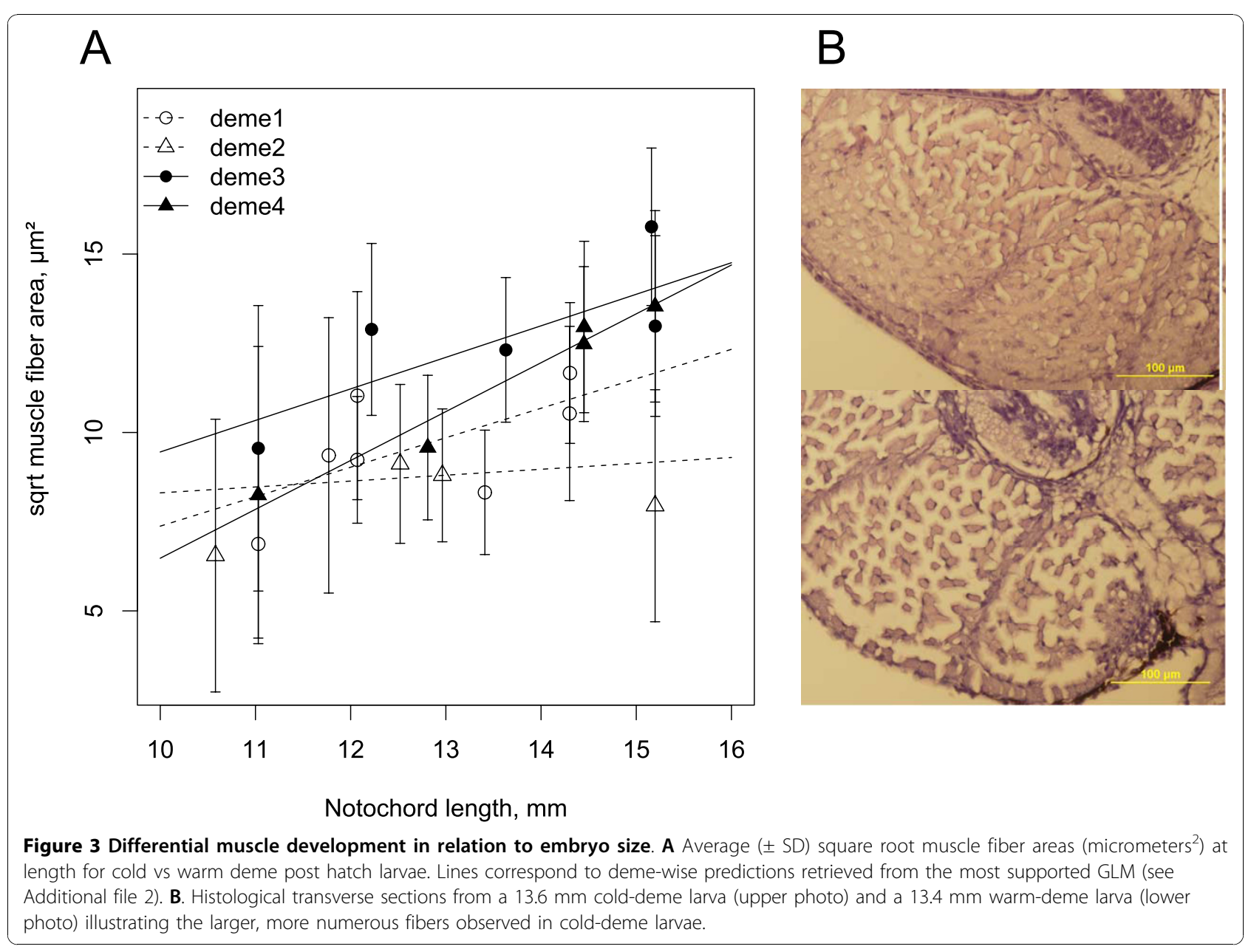

recently (90 years) established populations of chinook salmon (Oncorhynchus tshawytscha) in New Zealand. However, for the same populations embryo development rates did not differ [30]. As a consequence, due to temperature differences, the emergence dates could differ by up to six weeks between rivers. Hence, salmonids seem to have the potential to adapt to novel temperature regimes over fewer than 10 generations, even at spatial separation scales smaller than $10 \mathrm{~km}$. But it is not always the same traits that diverge, and therefore one cannot claim that adaptation is inevitable.

The patterns of differentiation in larval developmental rate and efficiency in relation to temperature in the Lesjaskogsvatnet grayling supports findings in a number of other studies; this include 1) the relationship between temperature environment and individual growth rate [32] and development rate [33,34], and 2) trade-offs between muscle growth and skeletal differentiation [22,35-37]. Taken together these results suggest that differentiation of individual growth, metabolism and development according to local environmental temperature is likely due to local adaptation. However, we also show that there are trade-offs among several of the musculoskeletal traits studied here. Rapid development of muscle comes at the cost of decreased development of functional structures. Thus, the adaptation of the various traits to different environmental conditions seems constrained by phenotypic (and probably genetic) correlations. Such intrinsic constraints, which probably are very common in biological systems, will, potentially, lead to a slower evolutionary response to environmental change and it will make predictions about the direction and speed of change difficult.

Temperatures in Nordic countries are expected to rise at higher-than-average rates over the coming decades [38], requiring Nordic lake fish populations, with no opportunity to migrate, to adapt (e.g., [33]). Recent studies from marine and terrestrial systems predict that the adaptive response to increase in temperature will be, firstly, that individuals will maximize growth and energy efficiency at the expense of ranges of thermal tolerance [39]. These studies also suggest that species at higher latitudes have broader thermal tolerance and are living in climates that are currently cooler than their physiological 


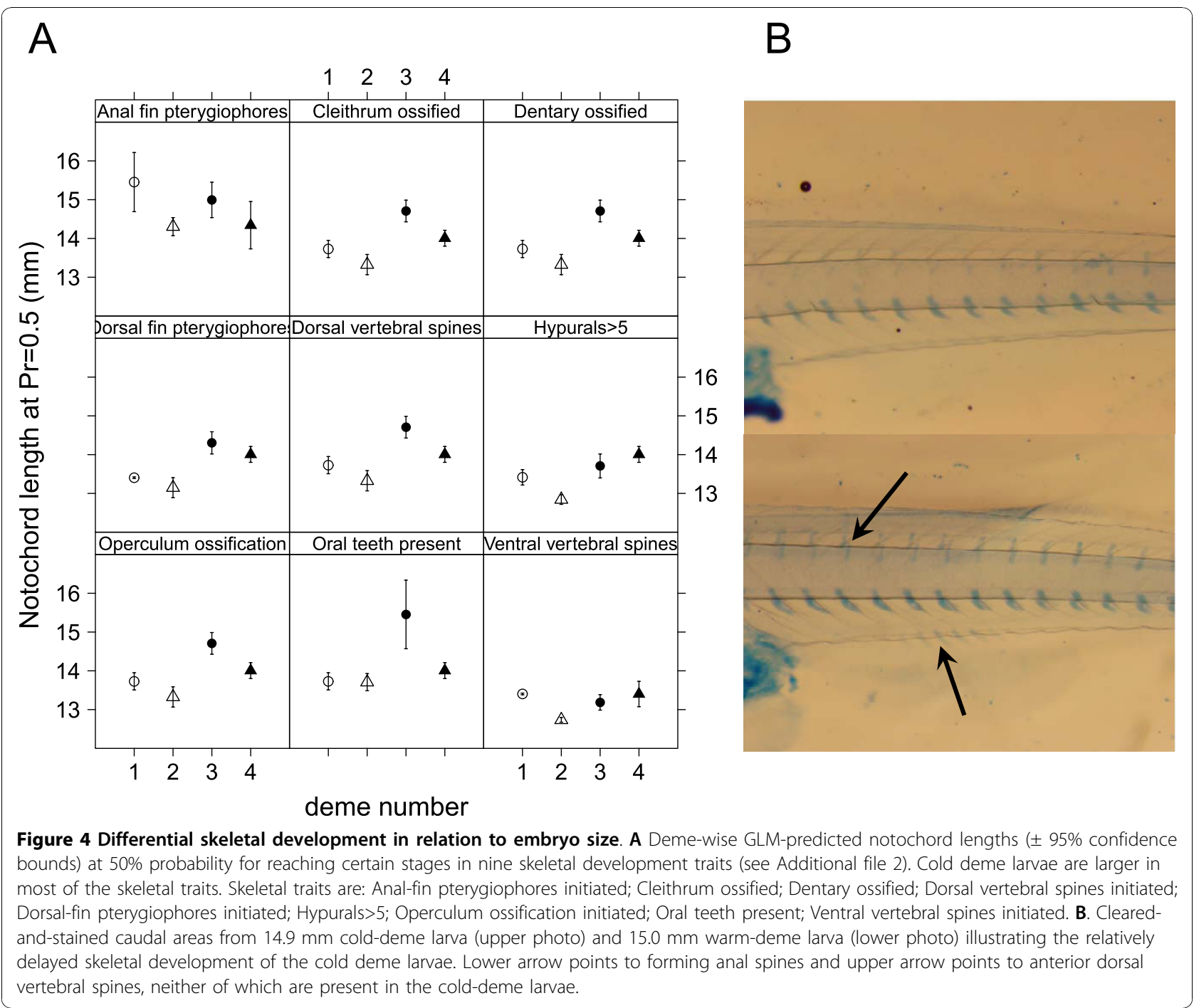

optima, and, as such, warming may even enhance their fitness [40]. For our specific grayling population under study, we found that the embryos were unable to successfully develop at temperatures above $12^{\circ} \mathrm{C}$ - indicating a narrow thermal tolerance. Grayling populations elsewhere in Europe clearly are able to develop successfully at these temperatures [41]. Field observations (unpublished information) of high clutch mortality following spring temperature spikes support the laboratory findings of mortality at high temperatures. In addition, both common-garden rearing experiments and long-term field observations of spawning temperatures indicate that grayling embryos cannot survive below $5^{\circ} \mathrm{C}$, further illustrating the narrow range of temperatures in which embryos of this grayling population can survive. Hence, will the grayling in Lesjaskogsvatnet be able to adapt to increasing temperatures? The apparent stenothermy suggests that phenotypic plasticity alone may not be sufficient for the grayling to cope, especially because we found no inter- or intra deme variation to successful development at $12^{\circ} \mathrm{C}$. Rather, the populations would need to evolve either a change in temperature responses or a change in spawning time (see Wedekind and Küng [42] for a grayling example). Indeed, a change in phenology is probably the most rapid way for a population to adapt to new climatic conditions $[4,43]$. There are very few reported examples that have demonstrated a genetic shift in thermal optima or thermal tolerance [43]. However, since the change in temperature will occur over decades, a gradual adaptation to the gradually changing seasonal water temperatures is likely through both change in phenology (spawning time) and change in temperature tolerance range. This mixture of changed phenology and gradual adaptation to slightly higher water temperatures is a likely evolutionary scenario since the water temperatures experienced by the embryos would be only slightly 
altered if the grayling start spawning earlier in spring. The prospects for an earlier spawning time is good as the current spawning time is very late (June) compared to other European populations (March-May [45]).

Previous studies of salmonids have shown that low temperatures may delay and prolong myogenic regulatory factor expression and muscle differentiation in the embryos [46]. Our result showing accelerated muscle, but delayed skeletal differentiation in low-temperature demes is indicative of a heterochronic evolutionary trade-off where resources are allocated competitively between growth and developmental processes in the musculoskeletal system. These types of internal tradeoffs have been hypothesized previously to affect evolutionary divergence patterns based on macroevolutionary patterns and cellular level mechanisms [22,35-37]. Since muscle and skeletal system development is linked to swimming performance in fishes, our data also suggest a connection to the emerging theory regarding performance trade-offs with fast intrinsic growth rates [22,23].

Previous reports show that isolated grayling populations derived from a single ancestral population rapidly adapt growth rates to local environmental conditions, even under strong influence of genetic drift $[14,47,48]$. We report here that for sympatric grayling demes in Lesjaskogsvatnet only a few weeks of temperaturespecific isolation per year seems to have been sufficient to cause genetic differentiation. Because we are working with evolution in wild populations, we cannot clearly distinguish whether temperature is directly and solely driving the divergence or whether there are indirect temperature effects (e.g., by interactions with the length of the growing season). However, other salmonid studies demonstrate direct temperature effects during embryogenesis that cause growth and muscle phenotype differences that persist into adulthood - even if the later ontogeny is expressed under similar environmental conditions [49]. In a compelling study on two Atlantic salmon populations in Scotland, Johnston and co-workers [50] demonstrated a higher yolk-to-body mass conversion efficiency and larger muscle fiber recruitment during embryogenesis in a cold-adapted population that was compared to a warm-adapted one. The coldadapted embryos did particularly well under thermal conditions similar to the one in their native stream indicating that the differences observed could be attributed to local adaptation. Our experimental design only allows for drawing inferences about genetic differentiation - and does not test for local adaptations directly. However, because of the replicated divergence pattern among demes observed in this study (parallelism), and also the direction of the divergence (as predicted from previous studies), we argue that the differences observed are largely due to local adaptations. The observed divergence is not likely to be primarily due to genetic drift because microsatellite-based $\mathrm{F}_{\mathrm{ST}}$-values were generally found to be smaller than $\mathrm{Q}_{\mathrm{ST}}$ values between deme pairs of differential temperature origin, and not different between deme pairs of similar temperature origin (Additional file 3). However, as pointed out in the methods section, one should interpret both the estimated $\mathrm{F}_{\mathrm{ST}}$ and $\mathrm{Q}_{\mathrm{ST}}$ values with caution as there are many sources of bias that may substantially have affected them both [51,52].

The ability to successfully adapt to new environments is assumed to be facilitated by large genetic variation [10]. Hence, bottleneck situations may therefore potentially affect a population's prospects for future adaptation. However, studies suggest that bottlenecked populations with low genetic diversity have a greater chance of avoiding extinction if they have opportunity for population expansion [53]. Annual gillnet catches of grayling in Lesjaskogsvatnet include up to 40,000 individuals, thus a post-colonization population expansion has occurred in this system. This opportunity for population expansion, following from the fact that the grayling colonized a system with available niches, may have played a major role in the observed divergence process and avoidance of local extinction. Even though gene flow most often is considered to hinder local adaptation [7] this is not necessarily always the case [54]. For instance, Garant et al. [55] predict that an intermediate level of gene flow will allow the greatest adaptive divergence. Gene flow among inbred demes may increase the evolvability of these demes, and as such enhance the short-term adaptation [10]. Detailed studies on not only among-deme dispersal patterns, but also offspring viability of between-deme crosses, would be needed to explore the consequences of gene flow for genetic variation and responses to selection.

\section{Conclusions}

This study provides evidence that natural selection is sufficiently powerful for temperate lake fish populations to adapt to novel temperature regimes within 22 generations, even under conditions with low genetic variation and under influence of gene flow. We also provide evidence pertinent to the IPCC-predicted global warming; namely that current genetic variation and constitution related to embryo development may not be sufficient to allow for adaptation to such a high temperature increase. However, by altering the spawning time the temperature during early development may change sufficiently little for adaptation to occur. These types of integrative studies, where genetic differentiation, ecological opportunities, and physiological limits are explicitly considered, will be essential in evaluating the capacity for populations to adapt to global warming. 


\section{Methods}

\section{Common-garden experiment}

Parents (7-15 males and 7-15 females from each stream) were sampled during the spawning run by using fyke nets in the streams. The warm streams were sampled during 2 to 6 June. The grayling in did not start spawning until 12 June in the cold streams and samples from these ones were retrieved during 12 to 14 June. They were assumed to represent random samples from their respective deme. Eggs and sperm were stripped from these ripe fishes and artificially fertilized in situ. Equal amounts of eggs $(0.2 \mathrm{~L})$ from each female were mixed together at deme level and this mixture was fertilized using a sperm mixture made up from equal amount from each male from the same deme. Fertilized eggs were kept at deme level in separate perforated plastic jars in $40 \mathrm{~L}$ containers and brought to the University of Oslo by car (total transportation time $6 \mathrm{~h}$ ). Here the fertilized eggs were held in small floating cages within $1 \times 1 \mathrm{~m}$ tanks and reared at two temperatures (8 and $12^{\circ} \mathrm{C}$ ) in the laboratory for 33 days, until yolk absorption (sensu [47]). Each deme was triplicated at each temperature. Dead eggs and larvae were registered and removed from the cages every day.

\section{Trait measurements}

Daily samples of ten individuals were taken haphazardly from the rearing units containing individual clutches and were preserved in $10 \%$ buffered formalin. Seventy individuals were selected for comparison of dry weights (5-7 individuals $\times 4$ demes $\times 3$ selected days). Samples were dried for 7 days at $60^{\circ} \mathrm{C}$. Weights were taken from 3 days post fertilisation (DPF, early egg), 20 DPF (prehatch; yolk and embryo were separated, dried, and weighed separately), and 31 DPF (larvae). Two of the largest specimens from each of the daily samples were taken for histological analysis ( $n=80 ; 20$ per deme). Largest specimens were selected because we reasoned that they were most representative of the healthy growth potential of the group. Average sizes were considered less informative as they are skewed by sickly or developmentally delayed individuals. All specimens were measured for notochord length $(N L)$, and then one size series was used for transverse sectioning and another size series for wholemount clearing and staining.

\section{Growth and developmental trait comparisons}

NIH Image software http://rsb.info.nih.gov/nih-image/ was used to obtain notochord length data from digital images taken of each sample $(n=5-10 /$ day/deme/ temperature).

For sections, samples were dehydrated through an alcohol series, then soaked in xylene $(12 \mathrm{~min} \times 3)$ and embedded with paraffin wax at $62^{\circ} \mathrm{C}(45 \min \times 3)$. Transverse sections of the body were taken from each sample at the anus $(8 \mu \mathrm{m})$. Sections were dried overnight, then rehydrated, stained with Haemotoxylin and Eosin-Y, dehydrated through an alcohol and xylene series, and mounted in Depex medium. Digital images of sections were captured and measurements were taken using NIH Image software. From muscle cross-sections, selected well-defined fiber areas $(n=10-112$ per individual) from a dorsolateral myotomal segment were measured by drawing an outline around individual fibers. Fibers from the central region of this myotomal segment were haphazardly selected. Wholemount clearing and staining allowed visualization of skeletal development. Formalin-fixed specimens were dehydrated through an alcohol series over one day, then trypsin digested and stained in Alcian Blue (cartilage) and Alizarin Red (bone) following standard protocols [56]. Each sample was examined under dissecting microscope for appearance of individual skeletal elements (cleithrum, dentary, gill arches, operculum, oral teeth), and meristic elements (number of dorsal or ventral vertebral arches, number of dorsal or anal fin pterygiophores, number of hypural elements in the caudal fin skeleton) were counted as they formed (Figure 4).

\section{Estimation of deme and group effects}

The $12^{\circ} \mathrm{C}$ treatments showed high mortality and high rate of malformations. There was a much higher percentage of gross developmental disorders at $12^{\circ} \mathrm{C}$ than at $8^{\circ} \mathrm{C}$ (61\% vs $\left.24 \%\right)$, with no obvious difference between cold and warm demes. As a consequence, statistical analyses were performed using data from the $8^{\circ} \mathrm{C}$ treatment only. The statistical analyses were performed using various generalized linear models (GLM) with link functions depending on the nature of the response variables (i.e., continuous or binomial). In general, all traits were analysed using notochord length and/or days post fertilization as predictor variables - in addition to either deme or temperature-group effects. The rationale for doing so is because the traits analysed change in value expression both as function of size relationships (NL) and of development time $(D P F)$. These variables were therefore included as covariates so as to study whether demes and/or temperature groups differentiated in covariateadjusted trait values - indicating genetic differentiation under the prevailing common-garden conditions. The covariate effects were modelled as fixed effects as eventual deme*covariate interaction effects were of particular interest, potentially indicating genetic differentiation in allometric and/or development trajectory relationships. In order to explicitly explore parallelism in trait responses for demes belonging to the same temperature 
group, deme effects were nested under temperature group effects. Hence, the core model for all analyses was:

$$
Y_{\mathrm{ijk}}=\alpha_{\mathrm{i}}+\alpha_{\mathrm{ij}}+\beta_{1 \mathrm{ij}} N L_{\mathrm{k}}+\beta_{2 \mathrm{ij}} D P F_{\mathrm{k}}+\beta_{3 \mathrm{ij}} D P F_{\mathrm{k}} N L_{\mathrm{k}}+\varepsilon_{\mathrm{ijk}}
$$

where $Y_{\mathrm{ij}}$ is the trait value for individual $\mathrm{k}$ in deme $\mathrm{j}$ $(\mathrm{j} \in\{1,2,3,4\})$ and group $\mathrm{i}(\mathrm{i} \in\{$ warm, cold $\}) . \alpha_{\mathrm{ij}}$ is the group effect for deme $i, \beta_{1 \mathrm{ij}}$ and $\beta_{2 \mathrm{ij}}$ are slope estimates for the effect of notochord length and time since fertilization, respectively. $\beta_{3 \mathrm{ij}}$ represents the interaction effect between the two covariates. In general, the residual variance was assumed to be distributed $N \sim(0,1)$ under the applied link function. For binomial traits the logit link function was applied. The most supported model structure was selected using Akaike Information Criterion [57]. All GLM analyses were performed using the glm procedure in $\mathrm{R}$ (version 2.10.1, [58]).

For extracting estimated notochord lengths (and corresponding standard errors) at a certain trait expression probability (i.e., $\operatorname{Pr}=0.5$ ) in some binomial skeletal traits the 'dose.p' procedure implemented in the $\mathrm{R}$ library MASS was applied to the selected trait-specific GLM models [59].

For the trait 'muscle fiber area' many fiber areas were measured per individual $(n=29 \pm 32$, mean \pm s.d. $)$. Hence, when modelling the effect of $N L$, group and deme for this trait the within-individual variance component had to be taken into account. This was done by adding ID as a random effect using a linear mixed model approach [60]. Due to the imbalanced data, the candidate models were fitted using the REML method implemented in the lme4 library in R [58]. The most supported model structure was chosen based on AIC.

In order to test for differentiation in notochord growth trajectories, a generalized additive model (GAM) [61] was used as no a priori parametric model of growth trajectories were available under the prevailing experimental conditions. This GAM approach enabled selection of the most parsimonious non-linear growth model by choosing the model involving the lowest degrees of freedom of the smoothing functions. The model selection was performed using generalized cross validation criterion [62] as implemented in the MGCV library in R (version 2.10.1 [58]).

A test of total dry weight of yolk and embryo as function of days since fertilisation and deme was conducted using ordinary two-way ANOVA with days since fertilisation coded as an ordinal variable with levels 3, 20 and 31 days since fertilization.

\section{$\mathrm{Q}_{\mathrm{ST}}$ and $\mathrm{F}_{\mathrm{ST}}$}

In order to assess the relative influence of genetic drift and natural selection to observed phenotypic differences between pairs of demes, we used a method recently suggested by Sæter et al. [63]. Using this approach, the range of potential $\mathrm{Q}_{\mathrm{ST}}$-estimates can be assessed even under conditions where the additive genetic components are not explicitly available. The authors argue that their method allows for $\mathrm{Q}_{\mathrm{ST}}$-estimation (or rather $\mathrm{P}_{\mathrm{ST}}$ estimation) even under ordinary field conditions where no information about family structure is available. Our experiment was conducted under common-garden conditions, but, owing to the lack of a family design, no estimates on additive genetic variance components could be derived. However, the between-population variance, or between-deme in our context, could be estimated. Following this approach, $\mathrm{Q}_{\mathrm{ST}}$ was estimated as:

$$
\mathrm{Q}_{\mathrm{ST}}=\frac{g \operatorname{var}(\mathrm{pop})}{g \operatorname{var}(\mathrm{pop}) h^{2} \operatorname{var}(\text { error })}
$$

where $g$ is the assumed additive genetic proportion of differences between populations, $h^{2}$ (narrow-sense heritability) is the assumed additive genetic proportion of differences between individuals within populations, var (pop) is the observed between-population variance component and var(error) is the observed within-population variance component. The ranges of likely $\mathrm{Q}_{\mathrm{ST}}$-estimates were simulated across different values of $g$ and $h^{2}$, with $\mathrm{g}$ ranging from 0.05 to 1 and $\mathrm{h}^{2}$ ranging from 0.05 to 0.8 . Owing to difficulties in assessing reliable residual variance components for binary traits ( 3 of the skeletal traits), we were able to estimate $\mathrm{Q}_{\mathrm{ST}}$ for 8 of the 11 traits involved in this study. The variance components were estimated from mixed models fitted using the lmer-procedure in the lme4 library in $\mathrm{R}$, version 2.10.1 [58]. In all models, fixed effects of $N L$ and/or $D P F$ were included and demes were coded as random effects.

Estimates of inter-population neutral genetic differentiation $\left(\mathrm{F}_{\mathrm{ST}}\right)$ and their $95 \%$ confidence intervals were obtained using the variance component approach (providing the $\mathrm{F}_{\mathrm{ST}}$ estimator theta) and bootstrapping of loci with program FSTAT version 2.9.3.2. http://www2.unil. ch/popgen/softwares/fstat.htm. In total, 18 polymorphic loci were used for this estimation (Additional file 4). The DNA used in the analysis was sampled from fin clips of 14-43 individuals from each deme during spawning time in 2004.

Inferences about whether a trait had diverged mainly due to natural selection were drawn, as suggested by Whitlock 2008 [52], based on whether the simulated $\mathrm{Q}_{\mathrm{ST}}$-values overlapped with the overall $\mathrm{F}_{\mathrm{ST}}$ probability distribution (i.e., 95\% confidence bound across all 18 loci). However, this $\mathrm{Q}_{\mathrm{ST}}-\mathrm{F}_{\mathrm{ST}}$ comparison is not to be considered a precise test for natural selection, but rather as a coarse assessment to explore if some traits and 
deme comparisons show consistent patterns of differences in the two metrics. Both the $\mathrm{F}_{\mathrm{ST}^{-}}$-values and $\mathrm{Q}_{\mathrm{ST}^{-}}$ values (in particular) estimated for this study are likely to be imprecise. The number of demes and number of loci involved were too low to produce reliable estimates (should have been $>10$ and $>20$, respectively [52]). The lack of a breeding design and possible common-garden experimental environment artefacts are sources of potential bias for the $\mathrm{Q}_{\mathrm{ST}}$ estimates.

\section{Additional material}

Additional file 1: Analysis of environmental differences between streams with particular attention to water temperature and consequences for timing of spawning and development

temperatures. A detailed description of a dynamic factor analysis (DFA) that utilize time-series data on water temperatures covering four years of data. The analysis groups the streams into cold and warm ones. This file includes Figure $\mathbf{A} \mathbf{1}$ where factor analysis part of the DFA is plotted for the different streams involved in the study. This file also includes estimates of spawning-time segregation using a generalized additive modelling approach where time and temperature sums are included as predictor variables. Includes Figure A2 showing the GAM model predictions for the timing of spawning.

Additional file 2: Parameter estimates and model fit statistics for the most supported models fitted to estimate group effects for 11 traits involved in the study (Table A1)

Additional file 3: Qst vs Fst comparisons between pairs of the four demes included in the study (Figure A3). The plots comprise sensitivity plots of $\mathrm{Q}_{\mathrm{ST}}$-values under various $\mathrm{g}$ and $\mathrm{h}^{2}$ settings. Left panels show simulations for muscle fiber area, middle panels for notochord length and right panels for the skeletal trait hypurals (as an example of skeletal trait). Horizontal dotted lines indicate the confidence interval for analogous $\mathrm{F}_{\mathrm{ST}}$-estimates and numbers provided in the figures correspond to mean $F_{S T}$ values and expected $Q_{S T}$ values, respectively. The expected $\mathrm{Q}_{\mathrm{ST}}$ corresponds to estimates where $\mathrm{g}=0.8$ and $\mathrm{h}^{2}=0.3$.

Additional file 4: Characteristics of microsatellite loci applied in the study. (Table A2).

\section{Acknowledgements}

We thank E. Harjunmaa for help with histology, Dimitar Serbezov for help with the experiments and sampling of embryos, and Claudia Junge for help with genotyping and $F_{S T}$ estimation. We also thank two anonymous reviewers for inputs that substantially improved the manuscript. The study has been financially supported by the Research Council of Norway (project \# 177728 N40 to LAV).

\section{Author details}

${ }^{1}$ Evolution and Development Unit, Institute of Biotechnology, University of Helsinki, P.O. Box 56 (Viikinkaari 9), 00014 Helsinki, Finland. ${ }^{2}$ Norwegian Institute for Water Research, Gaustadalléen 21, NO-0349 Oslo, Norway. ${ }^{3}$ Centre for Ecological and Evolutionary Synthesis, Department of Biology, University of Oslo, P. O. Box 1066 Blindern, NO-0316 Oslo, Norway. ${ }^{4}$ Hedmark University College, Campus Evenstad, NO-2418 Elverum, Norway. ${ }^{5}$ Department of Ecology and Evolution, Stony Brook University, Stony Brook, NY, USA. ${ }^{6}$ Department of Biology, University of Massachusetts Dartmouth, 285 Old Westport Road, North Dartmouth, MA, USA.

\section{Authors' contributions}

$\mathrm{KDK}, \mathrm{TOH}$, and LAV conceived the study. KDK, TOH, JJ, and FG contributed to the collection and analysis of the data. All co-authors contributed to refining the analyses, interpreting the results, and improving the manuscript. All co-authors read and approved the final manuscript.
Received: 16 March 2010 Accepted: 11 November 2010

Published: 11 November 2010

\section{References}

1. Hendry AP, Kinnison MT: The pace of modern life: measuring rates of contemporary microevolution. Evolution 1999, 53:1637-1653.

2. Rolshausen G, Segelbacher G, Hobson KA, Schaefer HM: Contemporary evolution of reproductive isolation and phenotypic divergence in sympatry along a migratory divide. Curr Biol 2009, 19:2097-2101.

3. Visser ME: Keeping up with a warming world; assessing the rate of adaptation to climate change. Proc $R$ Soc London B 2008, 275:649-659.

4. Bradshaw WE, Holzapfel CM: Climate change - Evolutionary response to rapid climate change. Science 2006, 312:1477-1478.

5. Hoffmann AA, Hallas RJ, Dean JA, Schiffer M: Low potential for climatic stress adaptation in a rainforest Drosophila species. Science 2003, 301:100-102.

6. Räsänen K, Hendry AP: Disentangling interactions between adaptive divergence and gene flow when ecology drives diversification. Ecol Lett 2008, 11:624-636.

7. Kawecki TJ, Ebert D: Conceptual issues in local adaptation. Ecol Lett 2004 7:1225-1241.

8. Hendry AP, Day T, Taylor EB: Population mixing and the adaptive divergence of quantitative traits in discrete populations: $A$ theoretical framework for empirical tests. Evolution 2001, 55:459-466.

9. Yeaman S, Guillaume F: Predicting adaptation under migration load: the role of genetic skew. Evolution 2009, 63:2926-2938.

10. Willi Y, Van Buskirk J, Hoffmann AA: Limits to the adaptive potential of small populations. Annu Rev Ecol Evol Syst 2006, 37:433-458.

11. Wake DB: What salamanders have taught us about evolution. Annu Rev Ecol Evol Syst 2009, 40:333-352.

12. Gienapp P, Teplitsky C, Alho JS, Mills JA, Merilä J: Climate change and evolution: disentangling environmental and genetic responses. $\mathrm{Mol}$ Ecol 2007, 17:167-178.

13. Lande R: Adaptation to an extraordinary environment by evolution of phenotypic plasticity and genetic assimilation. J Evol Biol 2009, 22:1435-1446.

14. Haugen $\mathrm{TO}$, Vøllestad LA: A century of life-history evolution in grayling. Genetica 2001, 112-113:475-491

15. Koskinen MT, Nilsson J, Veselov AJ, Potutkin AG, Ranta E, Primmer CR: Microsatellite data resolve phylogeographic patterns in European grayling, Thymallus thymallus, Salmonidae. Heredity 2002, 88:391-401.

16. Kristiansen $H$, Døving KB: The migration of spawning stocks of grayling, Thymallus thymallus, in Lake Mjøsa, Norway. Environ Biol Fishes 1996, 47:43-50.

17. Gregersen F, Haugen TO, Vøllestad LA: Contemporary egg size divergence among sympatric grayling demes with common ancestors. Ecol Freshw Fish 2008, 17:110-118.

18. Barson NJ, Haugen TO, Vøllestad LA, Primmer CR: Contemporary isolationby-distance, but not isolation-by-time, among demes of European grayling (Thymallus thymallus, Linnaeus) with recent common ancestors. Evolution 2008, 63:549-556.

19. Junge C, Vøllestad LA, Barson NJ, Haugen TO, Otero J, Sætre GP, Leder EH, Primmer CR: Strong gene flow and lack of stable population structure in the face of rapid adaptation to local temperature in a spring spawning salmonid, the European grayling (Thymallus thymallus). Heredity, in revision.

20. Hurst TP: Causes and consequences of winter mortality in fishes. J Fish Biol 2007, 71:315-345.

21. Johnston FD, Post JR, Mushens CJ, Stelfox JD, Paul AJ, Lajeunesse B: The demography of recovery of an overexploited bull trout, Salvelinus confluentus, population. Can J Fish Aquat Sci 2007, 64:113-126.

22. Arendt JD, Wilson DS: Population differences in the onset of cranial ossification in pumpkinseed (Lepomis gibbosus), a potential cost of rapid growth. Can J Fish Aquat Sci 2000, 57:351-356.

23. Billerbeck JM, Lankford TE, Conover DO: Evolution of intrinsic growth and energy acquisition rates. I. Trade-offs with swimming performance in Menidia menidia. Evolution 2001, 55:1863-1872.

24. Gagliano M, McCormick MI, Meekan MG: Survival against the odds: ontogenetic changes in selective pressure mediate growth-mortality trade-offs in a marine fish. Proc R Soc London B 2007, 274:1575-1582. 
25. Merilä J, Crnokrak P: Comparison of genetic differentiation at marker loci and quantitative traits. J Evol Biol 2001, 14:892-903.

26. Moore JS, Gow JL, Taylor EB, Hendry AP: Quantifying the constraining influence of gene flow on adaptive divergence in the lake-stream threespine stickleback system. Evolution 2007, 61:2015-2026.

27. Rosenblum EB: Convergent evolution and divergent selection: Lizards at the White Sands ecotone. Am Nat 2006, 167:1-15

28. Hendry AP, Wenburg JK, Bentzen P, Volk EC, Quinn TP: Rapid evolution of reproductive isolation in the wild: Evidence from introduced salmon. Science 2000, 290:516-518.

29. Hendry AP, Hensleigh JE, Reisenbichler RR: Incubation temperature, developmental biology, and the divergence of sockeye salmon (Oncorhynchus nerka) within Lake Washington. Can J Fish Aquat Sci 1998, 55:1387-1394.

30. Kinnison MT, Unwin MJ, Hershberger WK, Quinn TP: Egg size, fecundity, and development rate of two introduced New Zealand chinook salmon (Oncorhynchus tshawytscha) populations. Can J Fish Aquat Sci 1998, 55:1946-1953.

31. Kinnison MT, Unwin MJ, Quinn TP: Growth and salinity tolerance of juvenile chinook salmon (Oncorhynchus tshawytscha) from two introduced New Zealand populations. Can J Zool 1998, 76:2219-2226.

32. Billerbeck JM, Schultz ET, Conover DO: Adaptive variation in energy acquisition and allocation among latitudinal populations of the Atlantic silverside. Oecologia 2000, 122:210-219.

33. Jensen LF, Hansen MM, Pertoldi C, Holdensgaard G, Mensberg KLD, Loeschcke $V$ : Local adaptation in brown trout early life-history traits: implications for climate change adaptability. Proc R Soc London B 2008, 275:2859-2868.

34. Laugen AT, Laurila A, Rasanen K, Merila J: Latitudinal countergradient variation in the common frog (Rana temporaria) development rates evidence for local adaptation. J Evol Biol 2003, 16:996-1005.

35. Arendt JD: Adaptive intrinsic growth rates: An integration across taxa. Q Rev Biol 1997, 72:149-177.

36. Kavanagh KD: Embedded molecular switches, anticancer selection, and effects on ontogenetic rates: A hypothesis of developmental constraint on morphogenesis and evolution. Evolution 2003, 57:939-948.

37. Starck JM: Quantitative design of the skeleton in bird hatchlings - does tissue compartmentalization limit posthatching growth rates. J Morphol 1994, 222:113-131.

38. IPCC: Climate Change 2007: The Physical Science Basis. Contribution of Working Group I to the Fourth Assessment. Climate Change 2007: The Physical Science Basis. Contribution of Working Group I to the Fourth Assessment City: Cambridge University Press; 2007, 996.

39. Portner HO, Bock C, Knust R, Lannig G, Lucassen M, Mark FC, Sartoris FJ: Cod and climate in a latitudinal cline: physiological analyses of climate effects in marine fishes. Clim Res 2008, 37:253-270.

40. Deutsch CA, Tewksbury JJ, Huey RB, Sheldon KS, Ghalambor CK, Haak DC, Martin PR: Impacts of climate warming on terrestrial ectotherms across latitude. Proc Natl Acad Sci USA 2008, 105:6668-6672.

41. Humpesch UH: Inter- and intra-specific variation in hatching success and embryonic development of five species of salmonids and Thymallus thymallus. Arch Hydrobiol 1985, 104:129-144.

42. Wedekind C, Küng C: Shift of spawning season and effects of climate warming on developmental stages of a grayling (Salmonidae). Conserv Biol 2010.

43. Bradshaw WE, Holzapfel CM: Genetic response to rapid climate change: it's seasonal timing that matters. Mol Ecol 2008, 17:157-166.

44. Hill WG, Caballero A: Artificial selection experiments. Annu Rev Ecol Syst 1992, 23:287-310.

45. Northcote TG: Comparative biology and management of arctic and european grayling (Salmonidae, Thymallus). Rev Fish Biol Fish 1995, 5:141-194.

46. Xie SQ, Mason PS, Wilkes D, Goldspink G, Fauconneau B, Stickland NC: Lower environmental temperature delays and prolongs myogenic regulatory factor expression and muscle differentiation in rainbow trout (Oncorhynchus mykiss) embryos. Differentiation 2001, 68:106-114.

47. Haugen TO, Vøllestad LA: Population differences in early life-history traits in grayling. J Evol Biol 2000, 13:897-905

48. Koskinen MT, Haugen TO, Primmer CR: Contemporary fisherian life-history evolution in small salmonid populations. Nature 2002, 419:826-830.
49. Macqueen DJ, Robb DHF, Olsen T, Melstveit L, Paxton CGM, Johnston IA: Temperature until the 'eyed stage' of embryogenesis programmes the growth trajectory and muscle phenotype of adult Atlantic salmon. Biol Lett 2008, 4:294-298.

50. Johnston IA, McLay HA, Abercromby M, Robins D: Phenotypic plasticity of early myogenesis and satellite cell numbers in Atlantic salmon spawning in upland and lowland tributaries of a river system. J Exp Biol 2000, 203:2539-2552.

51. Hendry AP: Q(ST)> = not equal < F-ST? Trends Ecol Evol 2002, 17:502-502

52. Whitlock MC: Evolutionary inference from Q(ST). Mol Ecol 2008, 17:1885-1896.

53. Reznick DN, Ghalambor CK: The population ecology of contemporary adaptations: what empirical studies reveal about the conditions that promote adaptive evolution. Genetica 2001, 112:183-198

54. Nielsen EE, Hemmer-Hansen J, Poulsen NA, Loeschcke V, Moen T, Johansen T, Mittelholzer C, Taranger GL, Ogden R, Carvalho GR: Genomic signatures of local directional selection in a high gene flow marine organism; the Atlantic cod (Gadus morhua). BMC Evol Biol 2009, 9:276-287.

55. Garant D, Forde SE, Hendry AP: The multifarious effects of dispersal and gene flow on contemporary adaptation. Funct Ecol 2007, 21:434-443.

56. Potthoff T: Clearing and staining techniques. In Ontogeny and systematics of fishes (based on an international symposium dedicated to the memory of Elbert Halvor Ah/strom). Volume 1. Edited by: Moser HG, Richards WJ, Cohen DM, Fahay MP, Kendall AWJ, Richardson SL. [S.I.]: American Society of Ichthyologists and Herpetologists, Allen Press; 1984:35-37, [Lawrence K (Series Editor): Special Publication].

57. Burnham KP, Anderson DR: Model Selection and Inferences New York: Springer Verlag; 1998.

58. R Development Core Team: R: a language and environment for statistical computing. Book R: a language and environment for statistical computing. 2.10.1 edition. City: R Foundation for Statistical Computing; 2010.

59. Venables WN, Ripley BD: Modern Applied Statistics with S. 4 edition. Springer; 2002.

60. Zuur AF, leno EN, Walker N, Saveliev AA, Smith GM: Mixed Effects Models and Extensions in Ecology with R New York: Springer; 2009.

61. Hastie TJ, Tibshirani RJ: Generalized Additive Models London: Chapman \& Hall; 1990

62. Gu C, Wahba G: Minimizing GCV/GML scores with multiple smoothing parameters via the Newton method. Siam J Sci Statist Comp 1991, 12:383-398.

63. Saether SA, Fiske P, Kalas JA, Kuresoo A, Luigujoe L, Piertney SB, Sahlman T, Hoglund J: Inferring local adaptation from $\mathrm{Q}_{\mathrm{ST}}-\mathrm{F}_{\mathrm{ST}}$ comparisons: neutral genetic and quantitative trait variation in European populations of great snipe. J Evol Biol 2007, 20:1563-1576.

doi:10.1186/1471-2148-10-350

Cite this article as: Kavanagh et al:: Contemporary temperature-driven divergence in a Nordic freshwater fish under conditions commonly thought to hinder adaptation. BMC Evolutionary Biology 2010 10:350.

\section{Submit your next manuscript to BioMed Central and take full advantage of:}

- Convenient online submission

- Thorough peer review

- No space constraints or color figure charges

- Immediate publication on acceptance

- Inclusion in PubMed, CAS, Scopus and Google Scholar

- Research which is freely available for redistribution

Submit your manuscript at www.biomedcentral.com/submit
C Biomed Central 